\title{
Guanine nucleotides and acute renal failure
}

\author{
Joel M. Weinberg ${ }^{1}$ and Manjeri A. Venkatachalam ${ }^{2}$
}

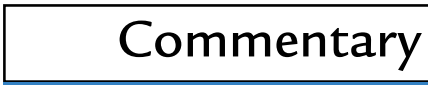

See related article, pages 1291-1298.

\author{
${ }^{1}$ Division of Nephrology, Department of Internal Medicine, University of Michigan and \\ Veteran's Administration Medical Center, Ann Arbor, Michigan, USA \\ ${ }^{2}$ Departments of Pathology and Medicine, The University of Texas Health Science Center at San Antonio, San Antonio, Texas, USA
}

Address correspondence to: Joel M. Weinberg, Nephrology Research, Room 1560, MSRB II, University of Michigan Medical Center, Ann Arbor, Michigan 48109-0676, USA. Phone: (734) 764-3157; Fax: (630) 604-5753; E-mail: wnberg@umich.edu.

J. Clin. Invest. 108:1279-1281 (2001). DOI:10.1172/JCI200114320.

Sustained ischemic and nephrotoxic acute renal failure (ARF) are characterized by overt damage to renal tubules, including cell necrosis. As a result, these processes have frequently been termed acute tubular necrosis, but this description has long been known to be misleading, as much of the tubule damage is sublethal, and the extent of necrotic cell death correlates imperfectly with the severity of organ dysfunction (1-3). Moreover, the organ dysfunction clearly includes a sustained hemodynamic abnormality during the maintenance phase, which most evidence indicates is persistent preglomerular arterial vasoconstriction (3). In this issue of the JCI, Kelly and coworkers report that pretreatment with guanosine ameliorates ischemic ARF in a murine model and that this improvement is associated with diminished apoptosis but not with changes in either necrosis or sublethal tubule cell damage (4). glycolysis as an alternative means of ATP generation in distal tubule segments and vascular cells when oxidative phosphorylation is blocked. At the tubule cell level, sublethal structural changes lead to shedding of apical microvilli and cast formation, causing tubule obstruction and decreased surface area for solute reabsorption, loss of tight junction integrity allowing back leak of glomerular filtrate, and impaired cell-cell and cell-substrate adhesion, which can result in loss of both viable and nonviable cells. Metabolic alterations set the stage for necrotic or apoptotic cell death.

At the vascular level, endothelial swelling, damage, and leakage lead to stasis and sludging, and abnormal production of vasoactive substances causes vasoconstriction. Expression of adhesion molecules on the surface of endothelial cells promotes localization of inflammatory cells, and cytokines and chemokines are released
Sublethal structural changes following renal ischemia can result in loss of both viable and non viable cells. Metabolic alterations set the stage for necrotic or apoptotic cell death.

\section{Cell and tissue damage in renal ischemia}

Current views of clamp ischemia models, such as those employed by Kelly et al. (4), postulate a sequence of interacting events (5-7). Severe metabolic disturbances with ATP depletion occur during the defined period of ischemia in both vascular and tubular cells. Early on, both cell types are protected from necrosis by their high levels of intracellular glycine, the $\mathrm{pH}$ drop that occurs during ischemia, and the availability of to further activate and recruit inflammatory cells. As a result, the effectiveness of reperfusion after the defined period of ischemia is reduced, and tubule cells are subjected to a continuing metabolic insult.

In murine models such as those used by Kelly et al. these vascular processes most strongly affect tubules in the outer stripe of the outer medulla because of the anatomy of the microcirculation to this area (6). Among the tubule segments traversing this region, the most extensive lethal cell injury develops in the proximal tubule. Although the medullary thick ascending limb is subject to rapid lethal damage in some settings (8), this nephron segment and other distal tubule cell types mainly develop sublethal changes in these clamp ischemia models and are the main sites at which activation of some stress signaling pathways can be detected (9). Susceptibility of proximal tubule cells to necrotic cell death is attributable to their low glycolytic capacity in vivo (7), which makes them prone to rapid, severe ATP depletion, opening of glycine-sensitive plasma membrane death channels (10), and necrotic cell death. These cells are also, by the same token, limited in their ability to activate ATP-dependent apoptotic cell death pathways (11). Distal tubule segments are more glycolytic (7) and, thus, have greater potential either to survive the insult or, when the insult is severe, to develop apoptosis (12).

\section{Effects of exogenous purines on the ischemic kidney}

Concentrations of ATP and GTP in proximal tubule cells can be transiently increased two- to threefold by providing extracellular nucleosides or nucleotides; the latter are catabolized to nucleosides that are then transported. ATP and GTP can be selectively manipulated in this fashion without affecting the other nucleotide (13). Studies of the beneficial effects of MgATP infusions on ischemic ARF were among the earliest to focus attention on metabolic approaches to modify cell injury states (14), but the multiplicity of effects of adenine nucleotides and their metabolites via cell surface receptors make the locus of their action unclear. The conversion of nucleosides to nucleotides is ATPdependent, albeit less so than de novo synthesis. For this reason, hypoxic cells 
without ongoing oxidative phosphorylation do not benefit from exogenous nucleoside treatment, with regard either to their ATP levels or to their ability to survive the injury (15).

Using combinations of purine synthesis inhibitors and either adenosine or guanosine, Dagher previously showed that selective ATP depletion in LLC-PK ${ }_{1}$ cells favors necrotic cell death, while GTP depletion produces apoptotic cell death (16). In the present work, Kelly et al. (4) extend this observation to show that promoting recovery of GTP with exogenous guanosine prevents the apoptosis that occurs after combined ATP and GTP depletion. Remarkably, a parallel to this behavior appears to occur in vivo, where both tubule cell apoptosis and organ dysfunction are ameliorated by guanosine.

\section{Guanosine and tubule cell survival}

Is the benefit of systemic guanosine in vivo attributable to primary effects on tubule cells, or to effects on any of the other potential target cells? Both alternatives are plausible. It has become clear that inflammatory effects play a major role in renal dysfunction after the primary insult in these models (5, $17,18)$. A previous study in the JCI reported that beneficial effects of ZVAD-fmk and IGF-1 on apoptosis and organ dysfunction during a similar model of ischemia/reperfusion are associated with reduced inflammatory cell infiltration (18). Kelly et al. (4) note that no cell surface guanosine receptors are known that could modulate inflammatory cells or cytokine/chemokine production, but, given the multiplicity of regulatory mechanisms that have been uncovered, such effects remain conceivable. Alternatively and very plausibly, in light of documented pathways, guanosine's salutary effects may be hemodynamically mediated. Augmentation of blood flow after ischemia, either by hemodilution, treatment with atriopeptin, or removal of the contralateral nonischemic kidney, can ameliorate ischemic ARF (6). The effects of atriopeptin and nitric oxide on renal blood flow in these models are mediated by cyclic GMP, and it has been reported that inhibition of cyclic GMP phosphodiesterase with zaprinast increases renal blood flow and ameliorates ARF, even when given a full day after ischemic injury (19). It is conceivable, although not tested in the current report, that increasing intracellular guanine nucleotides affects generation of cyclic GMP, thus improving blood flow in the injured organ.

In prior studies for which data are available, protective effects of antiinflammatory approaches and hemodynamic maneuvers, when applied early as in the present work, have usually been accompanied by a generalized reduction in the extent of cell injury. However, in their quantitative morphological analysis of guanosine-treated kidneys, Kelly et al. did not find this to be the case; only apoptosis was apparently affected (4). Additionally, guanosine was only effective if it was administered prior to the insult, suggesting a requirement for delivery to and metabolism by cells that could be less accessible or amenable to modulation after the insult. Such behavior might be expected more for tubule cells than circulating inflammatory or vascular cells. It is of interest in this regard that other agents reported to protect kidneys by preventing apoptosis also require early administration (18).

Assessment of apoptosis in settings such as ARF can be problematic. The commonly applied TUNEL assay, which was used in this study, can pick up necrotic cells as well as cells with repairable strand breaks $(20,21)$. However, Kelly et al. (4) have provided data on nuclear morphology suggesting reasonable specificity for the TUNEL assay under their conditions, supporting the conclusion that apoptosis has been preferentially modified. These authors have also gone to considerable lengths to identify the tubule cell types involved. Although this type of analysis is complicated by injury changes, the present study shows good retention of useful cell-specific markers. The number of apoptotic cells was reduced by guanosine in all tubule segments, but the proximal tubules also showed extensive necrosis and should have been severely compromised in any case. Perhaps, therefore, the apoptotic cell death blocked by guanosine is of greater significance in the distal tubules.

\section{Guanosine, apoptosis, and necrosis} Kelly et al. (4) suggest that the increased GTP induced by guanosine prevents apoptosis either by supporting nucleic acid synthesis or by activating small GTPases that are involved in the cytoskeleton and intracellular trafficking. These mechanisms are both likely, but other possibilities exist. GTP, for example, is an important energy transfer intermediate in the citric acid cycle in mitochondria, which are major intracellular triggers for apoptosis $(22,23)$. The cell culture models described in the present paper (4) and Dagher's earlier report (16) should allow this question to be definitively addressed in the future. Further studies will also have to determine whether apoptosis per se has a unique impact on additional tissue damage and organ dysfunction in these settings (18) or is simply an indicator of the severity of insult sustained by specific tubule segments with particularly important regulatory roles.

Human ischemic ARF is also characterized by an apparent dissociation between the extent of proximal tubule necrotic cell injury and organ dysfunction. There, relatively little necrosis is found despite severe organ dysfunction $(2,3)$, suggesting that sublethal changes in epithelial transport and barrier function are most important (3). However, involvement of other tubule segments, perhaps not so readily detected because of sampling limitations, has been suggested $(3,8)$. Such processes might include the lesion described in this paper.

1. Kreisberg, J.I., Bulger, R.E., Trump, B.F., and Nagle, R.B. 1976. Effects of transient hypotension on the structure and function of rat kidney. Virchows Arch. B Cell Pathol. 22:121-133.

2.Solez, K., Morel-Maroger, L., and Sraer, J. 1979. The morphology of "acute tubular necrosis" in man: analysis of 57 renal biopsies and a comparison with the glycerol model. Medicine (Baltimore). 58:362-376

3. Kwon, O., et al. 1999. Sodium reabsorption and distribution of $\mathrm{Na}^{+} / \mathrm{K}+$-ATPase during postischemic injury to the renal allograft. Kidney Int 55:963-975

4. Kelly, K.J., Plotkin, Z., and Dagher, P.C. 2001 Guanosine supplementation reduces apoptosis and protects renal function in the setting of ischemic injury. J. Clin. Invest. 108:1291-1298.

5. Sheridan, A.M., and Bonventre, J.V. 2000. Cell biology and molecular mechanisms of injury in ischemic acute renal failure. Curr. Opin. Nephrol. Hypertens. 9:427-434

6. Lieberthal, W., and Nigam, S.K. 1998. Acute renal failure. I. Relative importance of proximal vs. distal tubular injury. Am. J. Physiol. 275:F623-F631.

7. Weinberg, J.M. 1991. The cell biology of ischemic renal injury. Kidney Int. 39:476-500.

8. Brezis, M., and Rosen, S. 1995. Mechanisms of disease. Hypoxia of the renal medull: its implications for disease. N. Engl. J. Med. 332:647-655

9. di Mari, J.F., Davis, R., and Safirstein, R.L. 1999. MAPK activation determines renal epithelial cell survival during oxidative injury. Am. J. Physiol. 277:F195-F203.

10. Dong, Z., Patel, Y., Saikumar, P., Weinberg, J.M., and Venkatachalam, M.A. 1998. Development of 
porous defects in plasma membranes of ATPdepleted Madin-Darby canine kidney cells and its inhibition by glycine. Lab. Invest. 78:657-668.

11. Lieberthal, W., Menza, S.A., and Levine, J.S. 1998. Graded ATP depletion can cause necrosis or apoptosis of cultured mouse proximal tubular cells. Am. J. Physiol. 274:F315-F327.

12. Schumer, M., et al. 1992. Morphologic, biochemical, and molecular evidence of apoptosis during the reperfusion phase after brief periods of renal ischemia. Am. J. Pathol. 140:831-838.

13. Weinberg, J.M., Davis, J.A., Lawton, A., and Abarzua, M. 1988. Modulation of cell nucleotide levels of isolated kidney tubules. Am. J. Physiol. 254:F311-F322.

14. Siegel, N.J., et al. 1980. Enhanced recovery from acute renal failure by the postischemic infusion of adenine nucleotides and magnesium chloride in rats. Kidney Int. 17:338-349.

15. Weinberg, J.M., and Humes, H.D. 1986. Increases of cell ATP produced by exogenous adenine nucleotides in isolated rabbit kidney tubules. Am. J. Physiol. 250:F720-F733.

16. Dagher, P.C. 2000. Modeling ischemia in vitro: selective depletion of adenine and guanin nucleotide pools. Am. J. Physiol. Cell Physiol. 279:C1270-C1277.

17. Takada, M., Nadeau, K.C., Shaw, G.D., Marquette, K.A., and Tilney, N.L. 1997. The cytokineadhesion molecule cascade in ischemia/reperfusion injury of the rat kidney. Inhibition by a soluble P-selectin ligand. J. Clin. Invest. 99:2682-2690.

18. Daemen, M.A., et al. 1999. Inhibition of apoptosis induced by ischemia-reperfusion prevents inflammation. J. Clin. Invest. 104:541-549.
19. Guan, Z., Miller, S.B., and Greenwald, J.E. 1995 Zaprinast accelerates recovery from established acute renal failure in the rat. Kidney Int. 47:1569-1575.

20. Ohno, M., et al. 1998. "Apoptotic" myocytes in infarct area in rabbit hearts may be oncotic myocytes with DNA fragmentation: analysis by immunogold electron microscopy combined with In situ nick end-labeling. Circulation. 98:1422-1430.

21. Ueda, N., and Shah, S.V. 2000. Tubular cell damage in acute renal failure: apoptosis, necrosis, or both. Nephrol. Dial. Transplant. 15:318-323.

22. Kroemer, G., and Reed, J.C. 2000. Mitochondrial control of cell death. Nat. Med. 6:513-519.

23. Bernardi, P., Petronilli, V., Di Lisa, F., and Forte, M. 2001. A mitochondrial perspective on cell death. Trends Biochem. Sci. 26:112-117. 